\title{
Circle involute as an optimal scan path, minimizing acquisition time, in surface topography
}

\author{
Sergio Barbero ${ }^{1} \&$ Manuel Ritoré ${ }^{2}$ \\ ${ }^{1}$ Instituto de Óptica (CSIC), Serrano 121, Madrid 28006, Spain. \\ ${ }^{2}$ Departamento de Geometría y Topología (UGR), Granada 18071, Spain \\ E-mail: 1 sergio.barbero@csic.es. \\ E-mail: 2 ritore@ugr.es
}

\begin{abstract}
A relevant problem in point-by-point scanning surface topography is to find scanning paths minimizing the overall measurement time. We establish a rigorous mathematical framework that allows us to state, for the first time, that circle involutes are the best candidates for such scanning paths maintaining a prescribed measurement accuracy condition. We provide a quantitative analysis comparing time savings achieved by circle involutes with respect to three widely used scanning paths: 1) Archimedean spiral; 2) Equispaced concentric rings; 3) Equispaced parallel rectilinear lines. While time reduction with respect to the last two is significant, for the first is not the case, so indirectly supporting Archimedean spirals as a good choice too. We explain this fact by showing the close relationship between circle involutes and Archimedean spirals. Finally we perform some numerical simulations comparing circle involutes and Archimedean spirals within a disk (quasi-planar surfaces), assuming constant linear velocity of the measurement device and constant sampling frequency.
\end{abstract}

Keywords: point-by-point scanning surface topography; optimal scan path; circle involutes; Archimedean spirals Submitted to: Surf. Topogr.: Metrol. Prop. 


\section{Introduction}

Making faster surface topographic measurements has become a highly desired goal in several techniques, mainly because two reasons: first, it reduces the measurement errors in those cases where the surface measurement or the surface itself may change due to time perturbations; second, it reduces the measurement cost, something very valuable in industrial environments.

When surface topography is based on a point-by-point scanning procedure, for instance, scanning a contact probe or a non-contact optical measurement device, the overall total length of the scanning path strongly determines the total measurement time. In order to minimize the total length of the scanning path, Archimedean spiral curves have emerged as a reasonable choice. Archimedean spirals have been proposed in techniques as diverse as stylus profilometry [1], atomic force microscopy [2, 3], optical coherence tomography [4] or magnetic resonance imaging [5, 6]. However, apparently only heuristic arguments have lead to this choice; no rigorous mathematical argumentation has been used to sustain this decision and, even more importantly, to find another possible solutions.

Once the scanning path is selected, a second problem (although not necessarily independent) is to choose the measurement points (data distribution), which is fixed selecting the measurement device speed and the sampling frequency. When uniform and homogeneous data distribution is required, a good strategy could be to set constant linear velocity and sampling frequency along the scanning path [3, 4]. Although, this is not always technically possible because of constraints imposed by the scanning system (see, e.g. [7]).

Our goal was to establish a rigorous mathematical framework (based on calculus of variations and differential geometry) to derive which are the optimal sampling paths minimizing the overall measurement time, but at the same time maintaining a prescribed measurement accuracy condition. This framework allows us, first, to establish that circle involutes are the best candidates for such scanning paths and, second, to show the close relationship between circle involutes and Archimedean spirals, so indirectly supporting Archimedean spirals as a good choice too. We provide time savings of circle involutes with respect to two other widely used scanning paths: equispaced concentric rings and equispaced parallel rectilinear lines. Finally, we performed some numerical simulations comparing circle involutes and Archimedean spirals assuming constant linear velocity of the measurement device and constant sampling frequency (i.e. equal arc-length distance between measuring points).

We confined ourselves to quasi-planar surfaces (in the sense that elevation differences in surface geometry is a negligible factor when selecting the optimal scanning path) or of unknown geometry; one or the other is valid for the majority of cases in the aforementioned applications. 


\section{Statement of the problem}

To establish the problem, in mathematical terms, we assume some facts related to the time involved by the measurement procedure. Moreover, we set a measurement accuracy criteria.

As for the measuring time, we assume two physical assumptions, more or less realistic depending on the case: 1) the time required, by the measuring device, to acquire an elevation point measurement is constant along the scanning path $(t) ; 2$ ) the time involved by the measurement device while moving along the scanning path is proportional to the traveled distance (within surface geometry). Particularly, if the measurement device uses a constant linear velocity along the scanning path and constant sampling frequency, the overall measuring time would be $T=n(t+\tau)$, where $n$ is the number of measuring points and $\tau$ the time wasted by the scanning device to move between measuring points. In some cases, such as in MRI, $\tau$ is much larger than $t$, then stressing the role of minimizing the scanning time [8].

As for the measurement accuracy criteria, let's define a surface $S$ in a bounded domain $\Omega$ and a set of measuring points $g(i) \equiv(x(i), y(i), z(i)), i=1, \ldots, n$ defining a curve $C$; we impose that the distance of any point $P$ on $S$ to $C$ is less than a given magnitude $\epsilon$. This condition is essentially a density sampling criteria. In the particular case that the topography has a periodic structure (e.g. the surface nanometer structure of a synthetic material measured by atomic force microscopy), NyquistShannon theorem would establish the lower bound value for $\epsilon$ required to reconstruct the surface topography avoiding aliasing [9], although data sampling would be a critical factor too [10].

We establish that an optimal sampling, minimizing measuring time, is one solving

the following variational problem: given $\epsilon>0$, minimize the length $L(\gamma)$ of a Lipschitz curve $\gamma: I \rightarrow S$, where $I:=[0,1]$, such that the distance $d(p, \gamma(I))$ is less than or equal to $\epsilon$ for any point $p \in S$. In mathematical terms, we consider the class of curves

$$
\mathcal{A}_{\epsilon}:=\{\gamma: I \rightarrow S: \gamma \text { is lipschitz and } d(p, \gamma(I)) \leq \epsilon \quad \forall p \in S\},
$$

and we compute or approximate the quantity

$$
L_{\epsilon}:=\inf _{\gamma \in \mathcal{A}_{\epsilon}} L(\gamma)
$$

\section{Mathematical argumentation}

Using techniques of Calculus of Variations it is straightforward to prove, for fixed $\epsilon>0$, the existence of a Lipschitz curve $\gamma_{\epsilon}: I \rightarrow S$ minimizing the length in the class $\mathcal{A}_{\epsilon}$ (i.e., a solution to (2)). Uniqueness is not established and probably does not hold in general. Moreover, using purely mathematical methods we can show the existence of a closed set $I_{\epsilon} \subset I$ such that, outside $I_{\epsilon}$ and far from the boundary $\partial S$ of the circular disk, the curve $\gamma_{\epsilon}$ is composed of geodesics in $S$ (segments when $S$ is a disk) [11]. 
Geometrically, $t \in I_{\epsilon}$ if there exists a point $p \in S$ satisfying $d\left(p, \gamma_{\epsilon}(I)\right)=$ $d(p, \gamma(t))=\epsilon$. A remarkable case happens when $I_{\epsilon}=I$. These special solutions seem to be good candidates to minimizers since balls of radius $\epsilon$ can be placed in $S \backslash \gamma_{\epsilon}(I)$ touching any point of $\gamma_{\epsilon}(I)$. A particular case of these special solutions satisfying $I=I_{\epsilon}$ are the involutes, introduced by Christiaan Huygens in 1673, of closed circles [12].

When $S$ is the unit disk, we consider the circle of radius $r=\epsilon / \pi$ parameterized as $\alpha(s)=\left(r \cos \left(\frac{s}{r}\right), r \sin \left(\frac{s}{r}\right)\right)$ by arc-length. Its involute is the curve $\alpha(s)-s \alpha^{\prime}(s)$, obtained by moving a point at distance $s$ along the tangent line to the circle at the point $\alpha(s)$ in the direction of $-\alpha^{\prime}(s)$, so that the equation of the involute is

$$
\beta_{\epsilon}(s)=\alpha(s)-s \alpha^{\prime}(s)=\left(r \cos \left(\frac{s}{r}\right)+s \sin \left(\frac{s}{r}\right), r \sin \left(\frac{s}{r}\right)-s \cos \left(\frac{s}{r}\right)\right),
$$

whose length in the interval $\left[0, s_{0}\right]$ is equal to $r s_{0}^{2} / 2$. A disk of radius $\epsilon=\pi r$ is trapped between two consecutive turns of the involute and can be moved outside the curve always touching in two opposite points.

Figure 1 shows the circle involute of radius $r=\frac{\epsilon}{\pi}=0.01$.

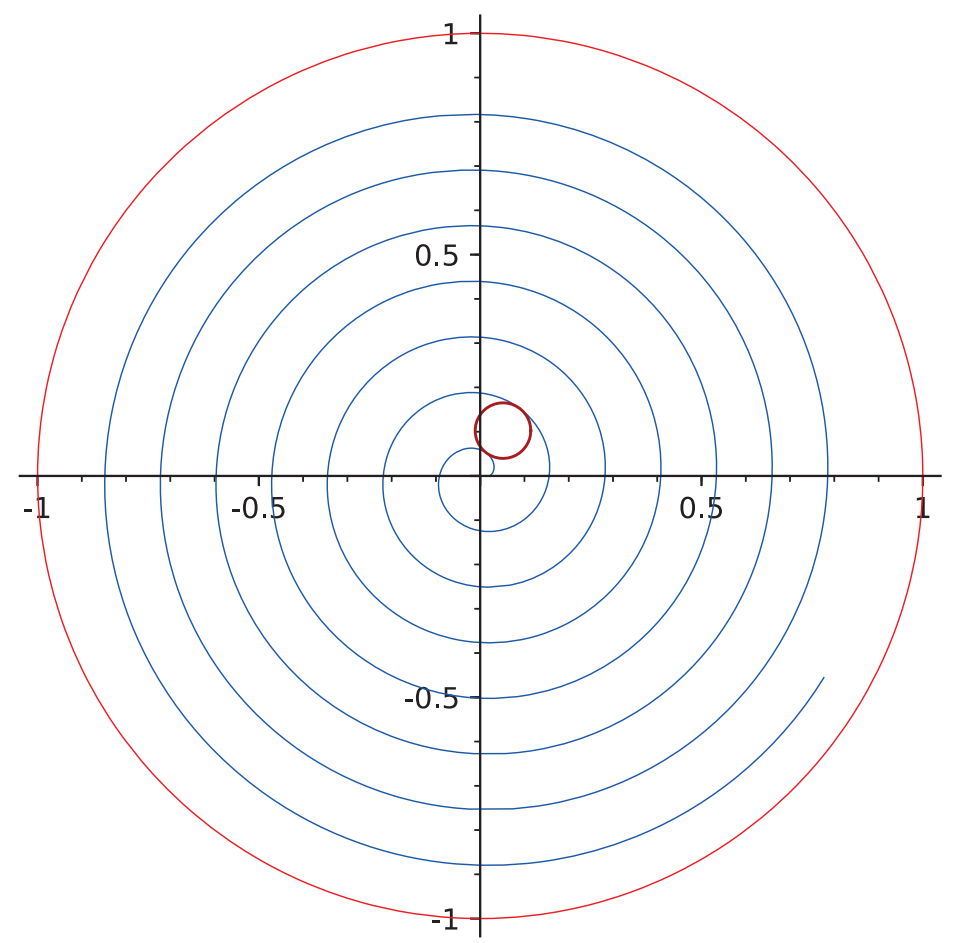

Figure 1. Involute in the unit disk of the circle of radius $r=\frac{\epsilon}{\pi}=0.01$.

\section{Outperformance of circle involutes over classical scanning paths}

We compared time involved by circle involutes and two conventional sampling paths defined within a circular disk. Say $\epsilon$ is the same as the one defined for the circle involute and $R$ is the radius of the circular disk. Then, we define the following scanning paths: 
Author guidelines for IOP Publishing journals in ${ }^{A} T_{E} X{ }_{2} \varepsilon$

(i) Curve composed of equispaced concentric rings joined by rectilinear segments.

$$
\begin{array}{r}
C^{\epsilon}:=\left\{(x, y): \sqrt{x^{2}+y^{2}}=(2 k-1) \epsilon, \quad k \in \mathbb{N}^{*}\right\} \cup \\
\left\{(x, y): y=0,(2 k-1) \epsilon<x<(2 k+1) \epsilon, \quad k \in \mathbb{N}^{*}\right\},
\end{array}
$$

where $k$ is an index setting the circle radius.

(ii) Curve composed of equispaced parallel rectilinear lines (in this case, vertical) joined by circle arcs contained in the boundary of the circular disk $(\partial S)$.

$$
\begin{gathered}
P^{\epsilon}:=\left\{(x, y): y=R-2(k-1) \epsilon,-\sqrt{R^{2}-y^{2}}<x<\sqrt{R^{2}-y^{2}}, \quad k \in \mathbb{N}^{*}\right\} \cup \\
\left\{(x, y): R-2(k-1) \epsilon>y>R-2 k \epsilon,-\sqrt{R^{2}-y^{2}}<x<\sqrt{R^{2}-y^{2}}, \quad k \in \mathbb{N}^{*}\right\},
\end{gathered}
$$

where $k$ is an index setting the $\mathrm{y}$-coordinate of the rectilinear lines.

Figure 2 and Figure 3 show the concentric ring and the parallel rectilinear lines sampling paths, respectively. In both figures the circle involute curve is also plotted for comparative purposes.

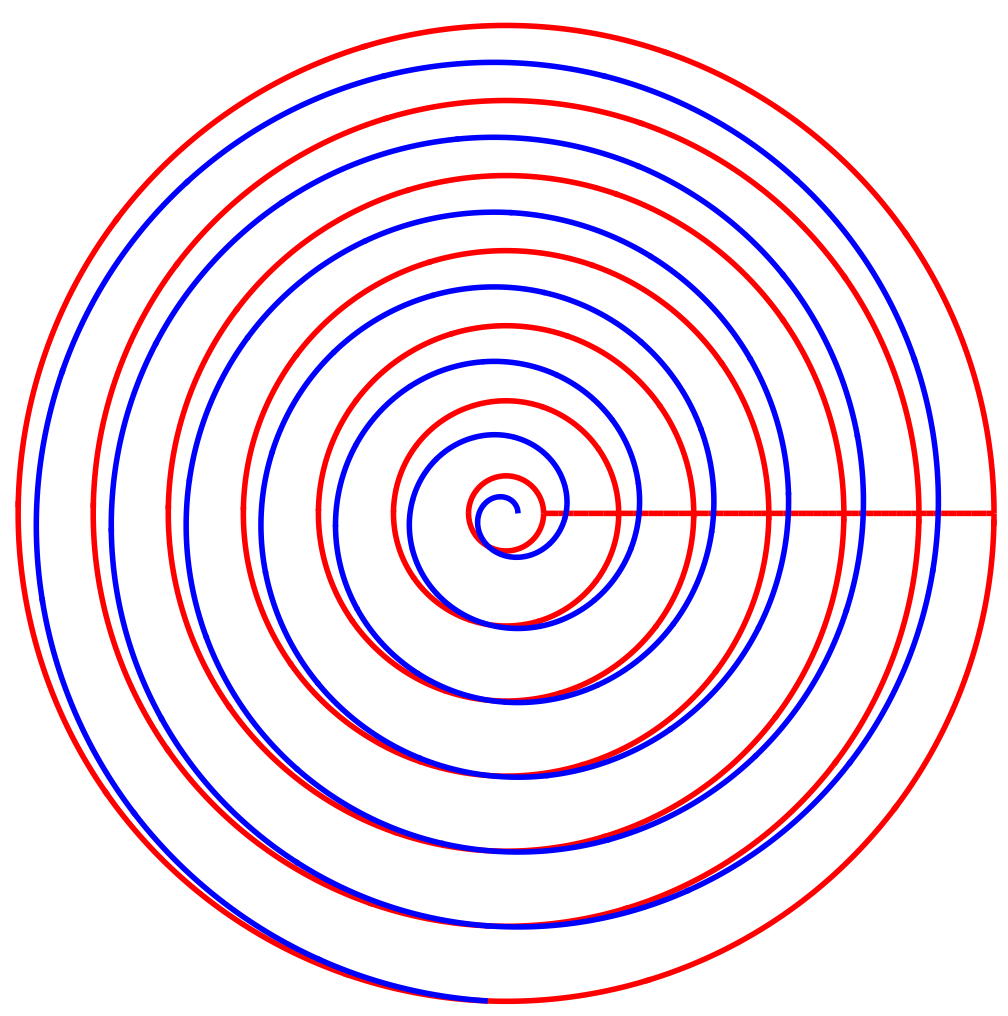

Figure 2. Circle involute (blue line) and concentric rings (red line) scanning paths.

We evaluated the differences between scanning times of these two conventional paths and the associated circle involute up to a radial coordinate (edge of the disk). Particularly, we computed scanning time reduction in percentages (\%) which, under the assumption of constant linear velocity, implies that length and time magnitudes are 


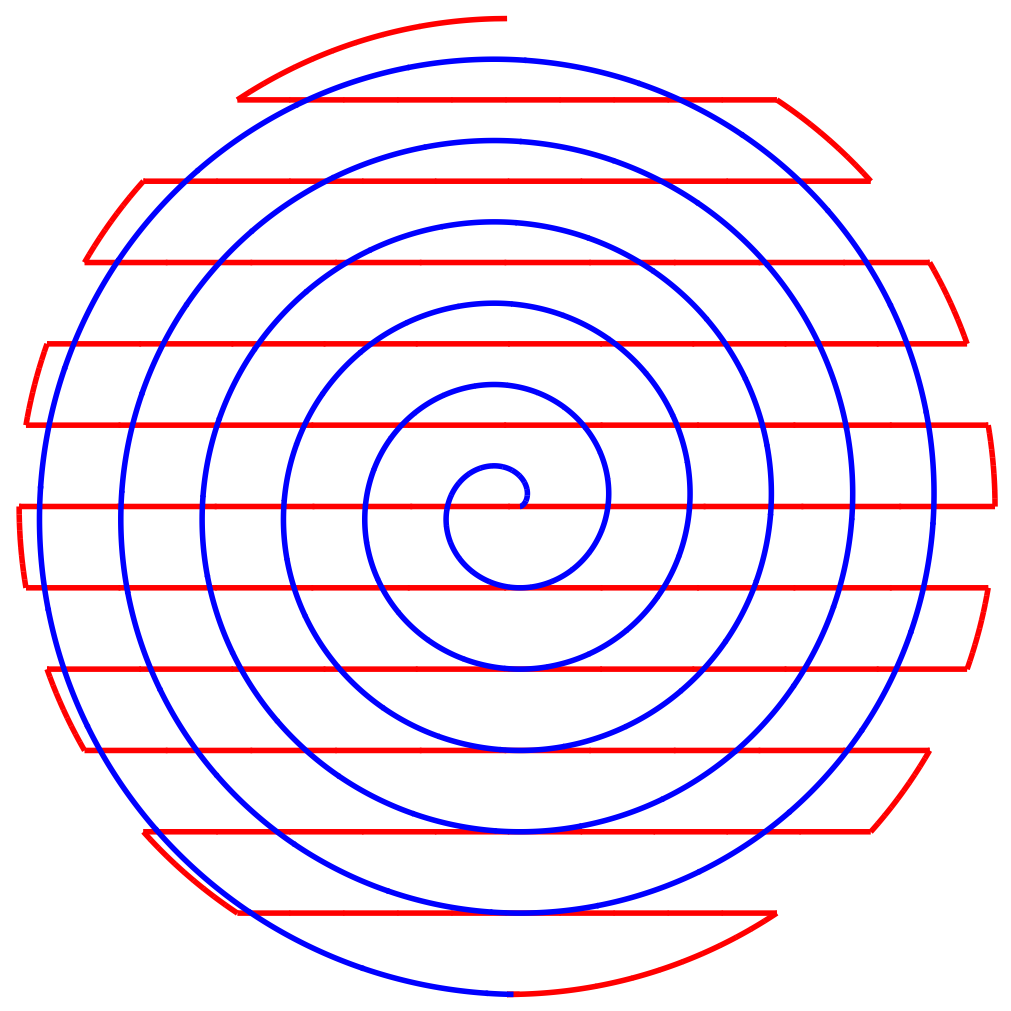

Figure 3. Circle involute (blue line) and parallel lines (red line) scanning paths.

equal. Figure 4 provides time savings as a function of the number of loops (\#Loops) obtained by the circle involute of radius $r=1$.

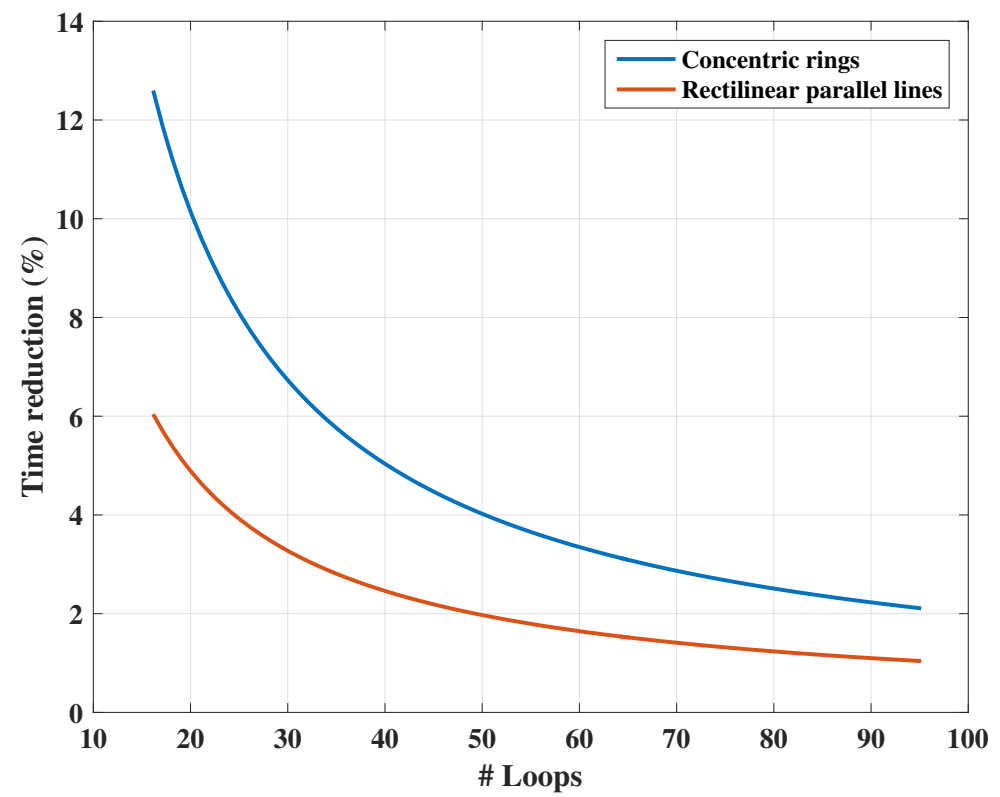

Figure 4. Measurement acquisition time saving (\%) of the circle involute with respect to the concentric rings and rectilinear parallel scanning paths as function of number of loops 
Figure 4 shows that for relatively moderate number of loops $(<20)$, time saved by the circle involute is around $5 \%$ and $10 \%$ with respect to the rectilinear parallel lines and concentric rings scanning paths, respectively. Time saving starts losing relative importance only with large number of loops. Although not showed in this graph, we found that the difference only goes below $0.1 \%$ when the number of turns is around 1000 and 2000 for the rectilinear parallel lines and concentric rings, respectively. This proves that in most cases time savings achieved by circle involutes would be potentially relevant with respect to the aforementioned conventional scanning paths.

\section{Relationship between circle involutes and Archimedean spirals}

The key property of circle involutes (in relation to our minimization problem) is the following one. Given a point $P$ of $S$, a geodesic passing through $P$ and cutting orthogonally the involute at one loop $Q_{1}$, also intersects orthogonally the circle involute at the adjacent loop $Q_{2}$, being the distance between points $Q_{1}$ and $Q_{2}$ always equal to $2 \epsilon: d\left(Q_{1}, Q_{2}\right)=2 \epsilon$. This ensures that the distance of any arbitrary point $P$ to the circle involute (radius $r=\frac{\epsilon}{\pi}$ ) is equal or less than $\epsilon$.

On the other hand we can construct an Archimedean spiral with a close, but different, property than that of the circle involute: the straight line starting at the center of the spiral and passing through $P$ intersects the spiral at adjacent loops at points $P_{1}$ and $P_{2}$ such that $d\left(P_{1}, P_{2}\right)=2 \epsilon$ :

$$
r=2 \epsilon\left(\frac{1+\theta}{2 \pi}\right) .
$$

However, this line is not orthogonal to the spiral curve. If we now construct the segment defining the distance from $P$ to the spiral (line orthogonal to the spiral at point $\left.T_{1}\right)$, the intersection of this line with the adjacent loop $\left(T_{2}\right)$ is not orthogonal. Besides, the distance between $T_{1}$ and $T_{2}$ is not equal to $2 \epsilon$. See Figure 5 for a better understanding of these geometrical properties.

The distance from $P$ to the spiral is less or equal than the minimum between distances $\min \left(d\left(P, P_{1}\right), d\left(P, P_{2}\right)\right)$. Now, considering that $2 \epsilon=d\left(P_{1}, P_{2}\right)=d\left(P_{1}, P\right)+$ $d\left(P_{2}, P\right)$, then $\min \left(d\left(P, P_{1}\right), d\left(P, P_{2}\right)\right) \leq \epsilon$. Therefore, the distance from $P$ to the spiral is equal or less than $\epsilon$. Now, the set of points, within the surface, that are exactly located at a distance $\epsilon$ from the measurement scanning curve is larger for the involute than for the spiral. Hence, the involute fills in the space more efficiently than the spiral. Anyhow, the closer $d\left(Q_{1}, Q_{2}\right)$ is to $d\left(T_{1}, T_{2}\right)$ the closer is the Archimedean spiral to the circle involute, and consequently to the optimal solution.

As in previous section we computed the differences between acquisition time (constant linear velocity) of spirals of Eq. (6) and circle involutes up to a radial coordinate (edge of the disk). Say $R$ is the radius of the disk to be measured. The number of loops of both curves is given by $\#$ Loops $=\frac{R}{2 \epsilon}$. 


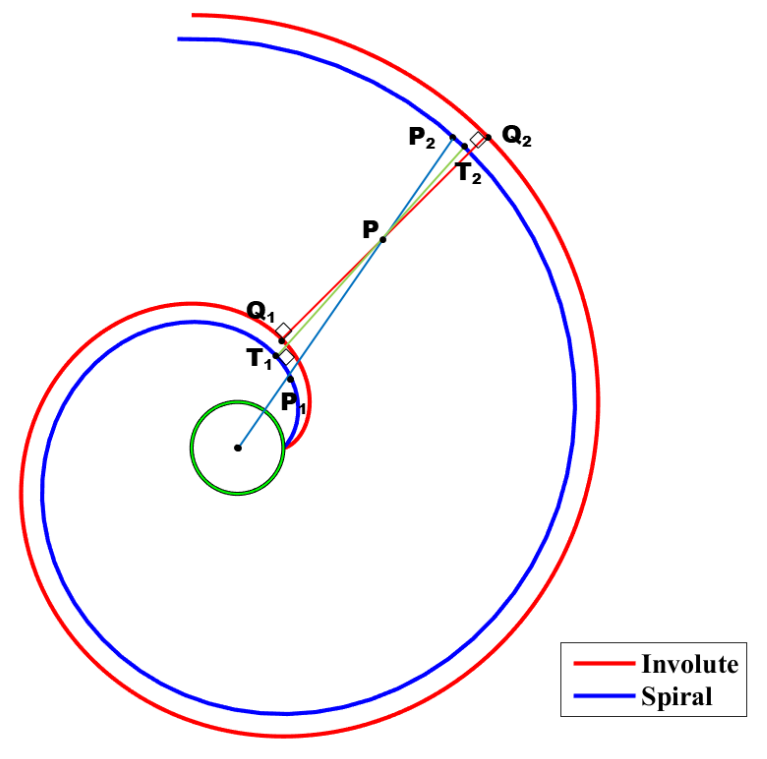

Figure 5. Comparison of distances between loops in the circle involute and the spiral and points $P$ of the disk.

Total length and therefore acquisition time of the spiral is always higher than that of the circle involute, though the difference increases slowly with increasing \#Loops .

Figure 6 shows time savings (\%) of the circle involute with respect to the spiral. Comparing results from Fig. 6 to that of Fig. 5 it is evident that differences between Archimedean spirals and circle involutes are much smaller than between circle involutes and other conventional scanning paths. Indeed, the relative difference converges uniformly to zero with \#Loops.

\section{Numerical results}

Once the scanning path is selected, a subsequent sampling problem is to choose the data distribution along that path, say $g(i), i=1, \ldots, n$. For the simulations of this section, we assumed that the measurement device moves with constant velocity along the scan path and that the sampling frequency is also constant. This implies that the set $g(i), i=1, \ldots, n$, meets the condition: $d(g(i), g(i+1))=k_{g}, i=1, \ldots, n-1$, being $k_{g}$ a constant. The value $k_{g}$ was chosen such that our accuracy criteria (the distance of any point $P$ on $S$ to $g(i), i=1, \ldots, n$, is less than a given magnitude $\epsilon$ ) is obeyed for all the the set. We compared $g(i), i=1, \ldots, n$, generated along circle involutes and Archimedean spirals of Eq. (6).

Our surface is quasi-planar and defined in a circular domain $S$, i.e. a disk of radius $R$. Of course, "any point $P$ on $S$ " has to be characterized numerically. We did it constructing a so-called Chebyshev net, i.e. a net of points forming a set of 


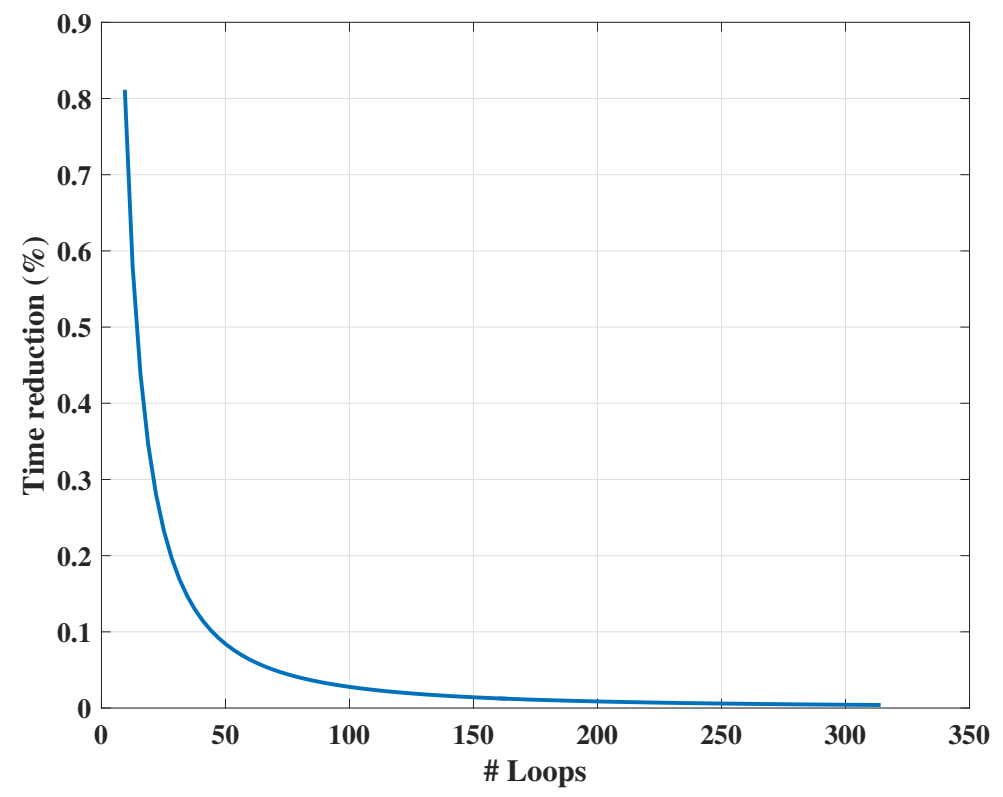

Figure 6. Measurement acquisition time saving (\%) of the circle involute with respect to the Archimedean spiral scanning path as function of number of loops

parallelograms such that all pairs of opposite sides have the same length [13]. This ensures that points are evenly distributed on the surface. For the case of the disk, the Chebyshev net is simply a regular cartesian net: $t(j), j=1, \ldots, m$. Due to computer memory limitations our Chebyshev net was limited to contain eight points between curve loops (following a line joining both loops).

Due to the numerical construction, once a measuring data density is selected, there are sampling frontier effects that may make the accuracy criteria fail close to the edge $\partial S$. For this reason, we redefine the scan path ending the involute and spiral at distance $\epsilon$ of $\partial S$, and subsequently continue the sampling with a circumference. Also, we omit points that are closer than $\epsilon$ to the disk center, which implies that the center of the lens is taken as an absolute reference.

We used several examples of disk sizes $R$ relative to $\epsilon: R=k_{n} \epsilon$, for different $k_{n}$. The sampling density was set to $k_{g}=\epsilon / 30$ in order to meet the accuracy criteria.

We characterized the accuracy criteria numerically as the minimum distances between sets $g(i), i=1, \ldots, n$ and $t(j), j=1, \ldots, m$. A particular example of disk sampling $\left(k_{n}=10\right)$ and $\epsilon=\pi$ is depicted in Figure 7. This figure shows the Chebyshev net and the measuring points for both scanning curves.

Figure 8 shows the minimum distances between sets $g(i), i=1, \ldots, n$ and $t(j), j=$ $1, \ldots, m$, denoted with: $d(t(j), g)_{j}$.

Besides our accuracy criteria, in certain cases it would desirable that the sampling points are distributed as homogeneous as possible [3]. We evaluated the homogeneity through the Root-Mean-Square and the standard deviation of the distances between 


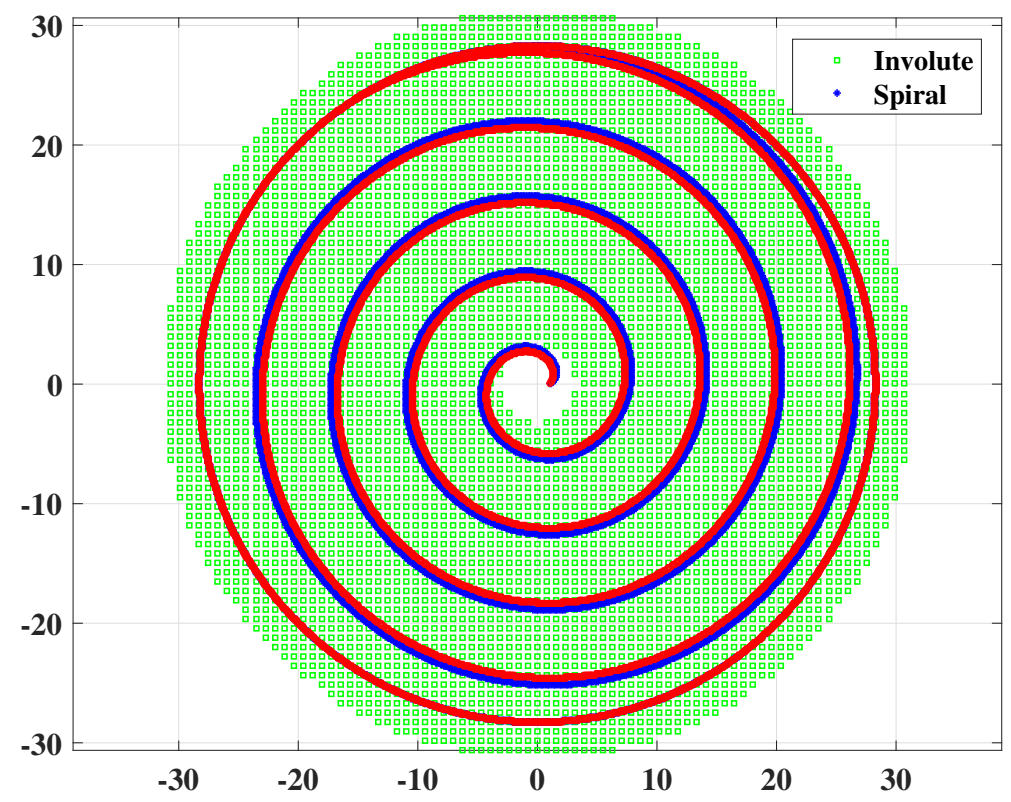

Figure 7. Chebyshev net (green circles) and measuring points along the spiral (red circles) and involute (blue stars).

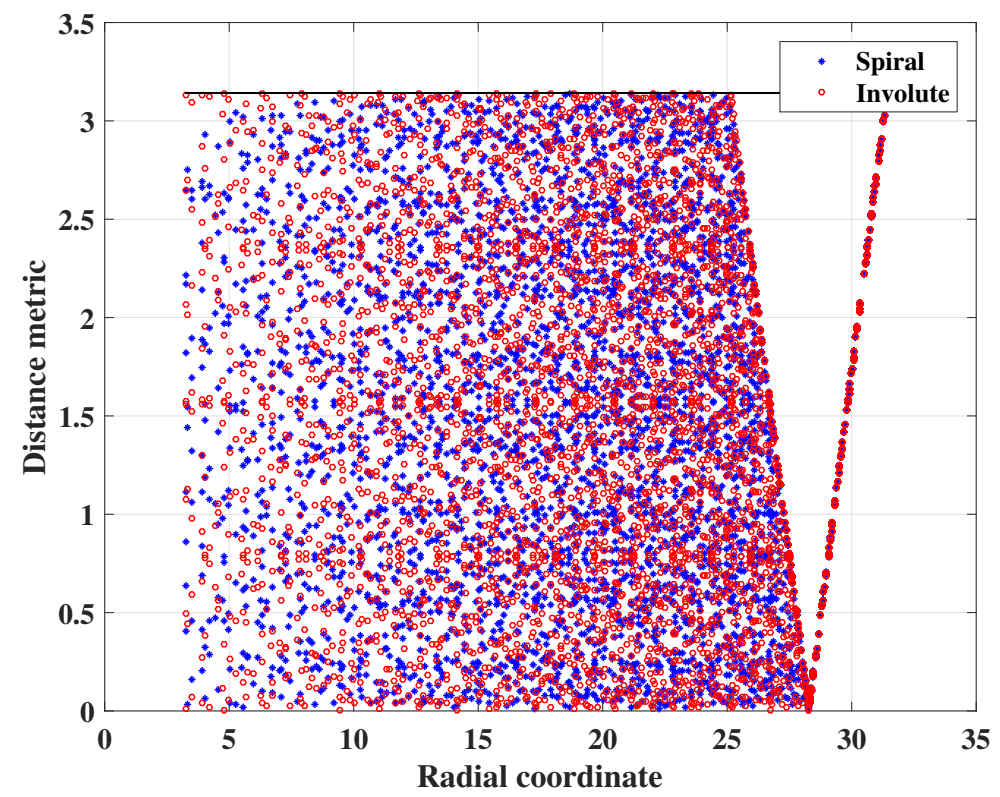

Figure 8. Minimum distances from points of the Chebyshev net to the measuring points along the spiral (blue stars) and involute (red circles).

sets $g(i), i=1, \ldots, n$ and $t(j), j=1, \ldots, m$.

$$
R M S=\sqrt{\frac{\sum_{j=1}^{m} d(t(j), g)_{j}^{2}}{m}}, \quad \sigma=\sqrt{\frac{\sum_{j=1}^{m}\left(d(t(j), g)_{j}-d(t, g)_{a}\right)^{2}}{m-1}},
$$

where $d(t, g)_{a}$ is the mean distance between sets $g(i), i=1, \ldots, n$ and $t(j), j=1, \ldots, m$.

Figure 9 provides RMS and $\sigma$ values as function of disk size $(\mathrm{mm})$. 


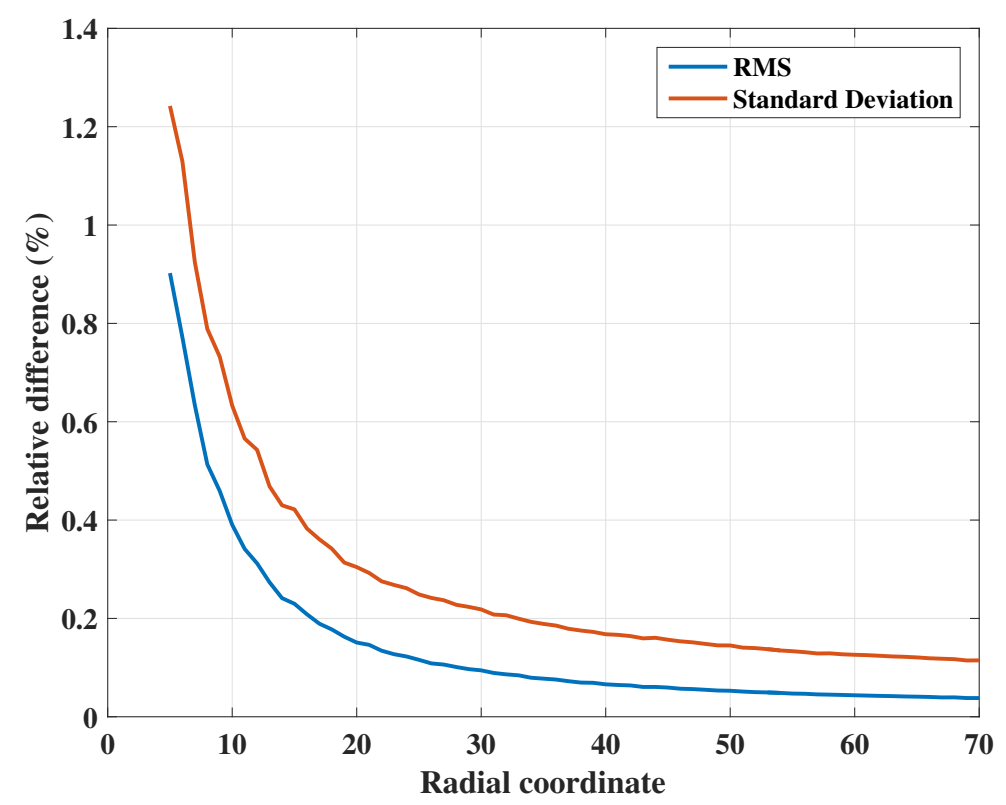

Figure 9. Relative difference (\%) between RMS and Standard Deviation of the distances between surface and measurement points using the spiral or the involute.

The results of Fig. 9 shows that the relative differences in, both RMS and $\sigma$, between the circle involute and the spiral is small and decreases asymptotically with the number of turns, although significantly the data distribution along the spiral is always distributed more homogeneously than that of the circle involute.

\section{Discussion}

The major contribution of this work is to sustain, by rigorous mathematical arguments, that circle involutes are optimal (in the sense of minimizing the overall measurement time) scanning paths in surface topography (when the surface domain is a circular disk) under certain specific premises (see section 2). This is, for our knowledge, the first time that circle involutes are proposed as a scanning path in point-by-point scanning topography. However, circle involutes are very similar to Archimedean spirals, specially when the number of turns is very large. Therefore, we indirectly showed the goodness of the widely spread strategy of choosing the Archimedean spiral as a scanning path.

We warn that one of the aforementioned premises, namely that the time involved by the measurement device while moving along the scanning path is proportional to the traveled distance (which intrinsically implies constant linear velocity of the scanning device) is, in several cases, a non realistic scenario. For instance, for some MRI techniques there are kinematic constraints associated to the speed and acceleration of the scanning device, which lead to a somewhat different mathematical optimal problem [7]. Additionally, the uniformly space samples along the scanning path is also not always desired or allowed, leading to a relevant sampling problem: the so-called variable density sampling along continuous trajectories [8, 10]. Taking into account all this makes the 
combined problem of selecting the scanning curve and the sampling points a highly complicate one. We just concentrated in solving the isolated problem of minimizing the scanning path length, which not implies ignoring the data sampling.

Although the mathematical results shown in section 3 are valid for any type of surface, our numerical results were applied for the case of quasi-planar surfaces; namely, those where the surface geometry in-the-large is not very relevant for selecting the optimal scanning path. This is the case for many of the applications mentioned in the introduction. However, this is not valid for many surfaces. In those cases, in general, there is no analytical equations for circles involutes and one has to resort to numerical solutions [11]. Also, distance computations have to be carried out within surface geometry through geodesic computations. We plan to address these cases in future work.

\section{References}

[1] M. Wieczorowski, "Spiral sampling as a fast way of data acquisition in surface topography," Int. J. Mach. Tool. Manu. 41(13), 2017-2022 (2001).

[2] I. A. Mahmood, and S. O. Reza, "Fast spiral-scan atomic force microscopy," Nanotechnology $\mathbf{2 0}(36), 365503$ (2009).

[3] D. Ziegler, T. R. Meyer, A. Amrein, A. L. Bertozzi, and P. D. Ashby, "Ideal scan path for high-speed Atomic Force Microscopy," IEEE-ASME. T. Mech. 22(1), 381-391 (2017).

[4] O. M. Carrasco-Zevallos, C. Viehland, B. Keller, R.P. McNabb, A.N. Kuo, and J.A. Izatt, "Constant linear velocity spiral scanning for near video rate 4D OCT ophthalmic and surgical imaging with isotropic transverse sampling," Biomed. Opt. Express 9(10), 5052-5070 (2018).

[5] C. B. Ahn, J. H. Kim, and Z. H. Cho, "High-speed spiral-scan echo planar NMR Imaging-I," IEEE Trans. Med. Imaging. 5(1), $2-7$ (1986).

[6] B. M. A. Delattre, R.M. Heidemann, L. A. Crowe, J. P. Valle, and J. N. Hyacinthe, "Spiral demystified," Magn. Reson. Imaging. 28(6), 862-881 (2010).

[7] C. Boyer, N. Chauffert, P. Ciuciu, J. Kahn, and P. Weiss, "On the generation of sampling schemes for magnetic resonance imaging," SIAM J. Imaging Sci. 9(4), 2039-2072 (2016).

[8] N. Chauffert, P. Ciuciu, J. Kahn, and P. Weiss, "Variable density sampling with continuous trajectories," SIAM J. Imaging Sci., 7(4), 1962-1992 (2014).

[9] A. V. Oppenheim, A. S. Willsky, and S. Hamid, Signals and systems, (Prentice-Hall, 1997).

[10] P. Jaming, F. Negreira, and J. L. Romero, "The Nyquist sampling rate for spiraling curves," eprint arXiv:1811.01771

[11] S. Barbero, M. Ritore "A geometric problem in surface topography," In preparation.

[12] D. J. Struik, Lectures on classical differential geometry, (Dover Publications, 1988).

[13] J. J. Stoker, Differential geometry, (Wiley-Interscience, 1969). 